\title{
Fluorescence laminar optical tomography for brain imaging: system implementation and performance evaluation
}

Mehdi Azimipour

Mahya Sheikhzadeh

Ryan Baumgartner

Patrick K. Cullen

Fred J. Helmstetter

Woo-Jin Chang

Ramin Pashaie 


\title{
Fluorescence laminar optical tomography for brain imaging: system implementation and performance evaluation
}

\author{
Mehdi Azimipour, ${ }^{\text {a, }}$ Mahya Sheikhzadeh, ${ }^{a, \dagger}$ Ryan Baumgartner, ${ }^{a}$ Patrick K. Cullen, ${ }^{b}$ Fred J. Helmstetter, ${ }^{b}$ \\ Woo-Jin Chang, ${ }^{c}$ and Ramin Pashaie ${ }^{\mathrm{a}, *}$ \\ aUniversity of Wisconsin-Milwaukee, Electrical and Computer Engineering Department, 3200 North Cramer Street, Milwaukee, \\ Wisconsin 53211, United States \\ bUniversity of Wisconsin-Milwaukee, Psychology Department, 2441 East Hartford Avenue, 207 Garland Hall, Milwaukee, Wisconsin 53211, \\ United States \\ 'University of Wisconsin-Milwaukee, Mechanical Engineering Department, 3200 North Cramer Street, Milwaukee, Wisconsin 53211, \\ United States
}

\begin{abstract}
We present our effort in implementing a fluorescence laminar optical tomography scanner which is specifically designed for noninvasive three-dimensional imaging of fluorescence proteins in the brains of small rodents. A laser beam, after passing through a cylindrical lens, scans the brain tissue from the surface while the emission signal is captured by the epi-fluorescence optics and is recorded using an electron multiplication CCD sensor. Image reconstruction algorithms are developed based on Monte Carlo simulation to model light-tissue interaction and generate the sensitivity matrices. To solve the inverse problem, we used the iterative simultaneous algebraic reconstruction technique. The performance of the developed system was evaluated by imaging microfabricated silicon microchannels embedded inside a substrate with optical properties close to the brain as a tissue phantom and ultimately by scanning brain tissue in vivo. Details of the hardware design and reconstruction algorithms are discussed and several experimental results are presented. The developed system can specifically facilitate neuroscience experiments where fluorescence imaging and molecular genetic methods are used to study the dynamics of the brain circuitries. @ 2017 Society of Photo-Optical Instrumentation Engineers (SPIE) [DOI: 10.1117/1.JBO.22 .1.016003]
\end{abstract}

Keywords: optogenetics; fluorescence optical tomography; fluorescent protein; optical imaging; Monte Carlo.

Paper 160452RR received Jun. 29, 2016; accepted for publication Dec. 12, 2016; published online Jan. 5, 2017.

\section{Introduction}

Optical imaging provides mechanisms to interrogate the dynamics of data processing in large-scaled networks of the brain. This information can then be used to better understand the causes and circuitries involved in neurological and psychiatric diseases. These methods allow for none or minimally invasive monitoring of functional interactions and metabolic changes in brain cells including hemoglobin oxygenation, action potential generation, or measuring ion concentrations, with reasonable temporal resolution either via intrinsic or extrinsic contrast changes in absorption, fluorescence, or scattering parameters of the tissue. ${ }^{1}$

One challenge in imaging biological tissue with optics is the multiple scattering of photons which distorts the propagation of the waves through the tissue, causing loss of directionality. Scattering has significant effect on the performance of optical imaging systems to the point that in brain imaging experiments the main variables quantifying the scanner's performance (e.g., the penetration depth and achievable imaging resolution) are closely determined by the scattering parameters of the tissue. ${ }^{2}$

In several optical imaging modalities, such as laser scanning microscopy (LSM) methods including confocal ${ }^{3}$ and twophoton microscopy, ${ }^{4}$ the effect of scattering is intentionally suppressed by minimizing the contribution of scattered photons in image formation. While high-resolution images are obtained by

*Address all correspondence to: Ramin Pashaie, E-mail: pashaie@uwm.edu

†These authors contributed equally. these methods, the penetration depth is minimum and imaging is limited to only superficial layers. A different class of optical imaging techniques, such as diffuse optical tomography (DOT), was also studied based on the idea of making use of multiple scattered photons in an effective way to reach and image deeper objects even at the cost of sacrificing the achievable resolution. ${ }^{2}$

Laminar optical tomography (LOT) ${ }^{5}$ was developed as an imaging modality that fills the gap between these two discernable classes of optical imaging methods. LOT provides a noncontact imaging technique to probe superficial parts of tissue offering better resolution (100 to $200 \mu \mathrm{m}$ ) than DOT and larger penetration depth (up to $2 \mathrm{~mm}$ ) compared to LSM techniques. Since LOT can image a wide range of parameters, including the absorption, scattering, and fluorescence contrast, over a large field of view (FOV) and with high acquisition speeds, this method is suitable for imaging relatively thin biological samples such as retina, skin, endothelial tissues, and more interestingly cortical regions of the brain. ${ }^{6}$ The main objective of this study is to explore the potential of the LOT method for fluorescence imaging of the cortical tissue in small rodents.

The advent of molecular genetic techniques has opened new horizons in the study of the brain complex and interconnected networks. With the use of genetic techniques, we can alter or manipulate the functionality of different cell-types in such large networks and step-by-step decipher and understand the role of each cell population in the overall collective function of

$1083-3668 / 2017 / \$ 25.00$ @ 2017 SPIE 
the networks. A good example of such techniques is the method currently known as optogenetics. ${ }^{7,8}$ In this technique, specific cell populations in the brain are genetically targeted to produce light-gated proteins which function as ion channels and pumps. By introducing such proteins in certain neurons, we can increase or suppress the activity in such cells just by exposing the cells to appropriate wavelengths. Recently published reports have proved that the method has significant potential in the study of central and peripheral nervous systems in different species. ${ }^{7,8}$

Obviously, proper expression of such light-activated proteins following viral gene delivery in targeted cells is essential for any successful in vivo optogenetic experiments. A common practice in assessing the level of success in gene delivery is the use of fluorescence proteins which are coexpressed in target cells. The existence and spatial spread of fluorescence proteins, used as genetically encoded biomarkers, indirectly confirms the expression of optogenetic tools. This information helps to better position and implant an optical fiber which guides the laser power to the transfected region and also determines the intensity of laser pulses needed to effectively stimulate the cells. ${ }^{9}$ The development of a noninvasive fluorescence tomography scanner is highly desired to assess the light sensitization process prior to running any optogenetic experiment, without sacrificing the animals. The results provided in this paper prove that an appropriately designed fluorescence laminar optical tomography (FLOT) scanner can serve this purpose and provide reasonably accurate three-dimensional (3-D) images of optogenetic protein expression in the cortical regions of the brain tissue.

LOT was first implemented in $2004^{5}$ as a mesoscopic imaging technique, which incorporates the measurement geometry of DOT with a microscopy-based setup. LOT performs depthresolved 3-D functional imaging, offering 100 to $200 \mu \mathrm{m}$ resolution and imaging depth up to $2 \mathrm{~mm}$. It was initially developed for multispectral imaging of the rat brain and results were published in $2007 .^{10,11}$ Since then, several advances have been made to the technique, including the integration of the system with other modalities such as optical coherence tomography (OCT). ${ }^{12,13}$

\section{Materials and Methods}

\subsection{Laminar Optical Tomography}

In a typical LOT system, a noncontact laser scanning setup ${ }^{5,10}$ similar to confocal microscopy is used to raster scan the tissue by a focused beam of light directed by a pair of moving galvanometer mirrors. The returning light is detected from both the focal point of the scanning beam and at a series of increasing distances $(0.0$ to $1.6 \mathrm{~mm})$ from the beam's focal point, allowing the superficial and deeper structures to be distinguished for 3-D image reconstruction, see Fig. 1.

In the fluorescence LOT scanner, the excitation light can be delivered in the form of scanning point source ${ }^{5}$ or sweeping line illumination. ${ }^{13}$ In point illumination, to acquire high-resolution data, fast photodetectors are required to reduce the scan time. The method of choice in this project was the line illumination and to reduce the scan time, an electron multiplication CCD camera was used to acquire data along up to nine detector arrays ( 0.0 to $1.6 \mathrm{~mm})$ parallel to the illumination pattern.

\subsection{Experimental Setup}

The schematic of the implemented FLOT system is shown in Fig. 2. A single-mode fiber-coupled blue laser (LP450-SF15,

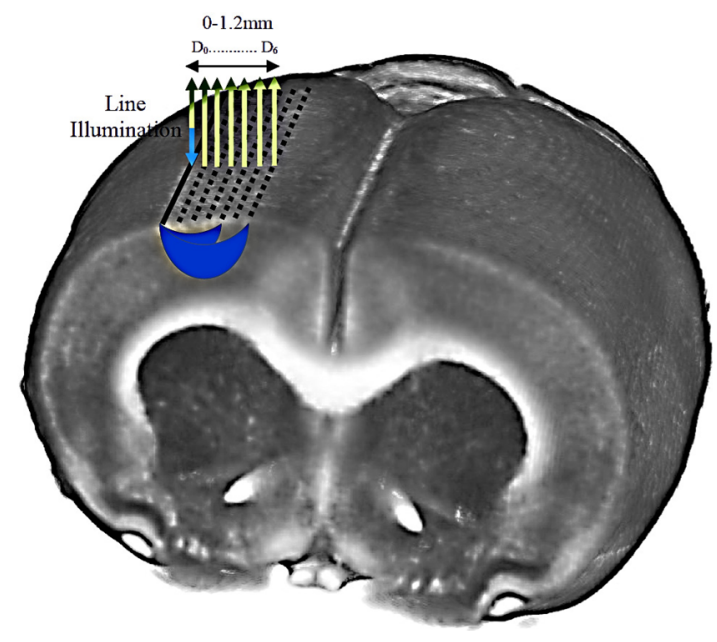

Fig. 1 FLOT measurement geometry and source-detector configuration in a line pattern illumination. The emitted fluorescent signal is collected from up to nine detector arrays parallel to the line illumination with separations of 0.0 to $1.6 \mathrm{~mm}$. Seven source-detector pairs with the separation between 0.0 to $1.2 \mathrm{~mm}$ are shown.

450-nm single-mode pigtailed laser diode, Thorlabs Inc., Newton, New Jersey) is used to achieve a sharp focus when delivering the excitation light. The light from the blue laser is collimated (F810SMA-635, Thorlabs Inc., Newton, New Jersey) and then passed through a cylindrical lens (LJ1695RM, Thorlabs Inc., Newton, New Jersey) to obtain the line-field illumination with a full line-width at the half maximum of $42 \mu \mathrm{m}$ on the sample. Next, the beam is passed through a dichroic mirror (FF440/520-Di01, Semrock, Rochester, New York). Wavelengths shorter than a threshold (excitation light) pass through the dichroic mirror, whereas longer wavelengths (emission light) are reflected toward the imaging components of the system. After passing through the dichroic mirror and before reaching the scan lens, an achromatic doublet lens (AC254-060-A-ML, Thorlabs Inc., Newton, New Jersey) with a focal length of $f_{2}=60 \mathrm{~mm}$ is placed in a way that the distance between the cylindrical and achromatic lens is $f_{1}+f_{2}$. Then the excitation light hits on a pair of moving $X$ and $Y$ galvanometer mirrors (GVS212, Thorlabs Inc., Newton, New Jersey). The galvanometer mirrors are controlled by the computer software prepared under LabVIEW (National Instruments, Austin, Texas) to steer the collimated beam through a scan lens. The scan lens (LSM03-VIS, Thorlabs Inc., Newton, Austin) focuses the line beam on the surface of the tissue to scan the predefined FOV by converting the angular changes on one of the galvanometer mirrors into a horizontal translation of the scanning line.

The emitted light produced by the fluorophores inside the tissue is collected by the same scan lens and descanned by the galvanometer mirrors. To this point, the excitation and detection paths overlap. Nonetheless, when the returning light is propagated backward in the system and reaches the dichroic mirror, only the emission wavelengths are reflected toward the light detection components of the system. To completely remove the unwanted wavelengths, a narrow-band green emission filter is used in front of the detector. The light entering the optical filter is a converging beam. Therefore, to eliminate problems associated with placing an optical filter in front of a focusing beam, such as the leakage from the emission filter and 
(a)

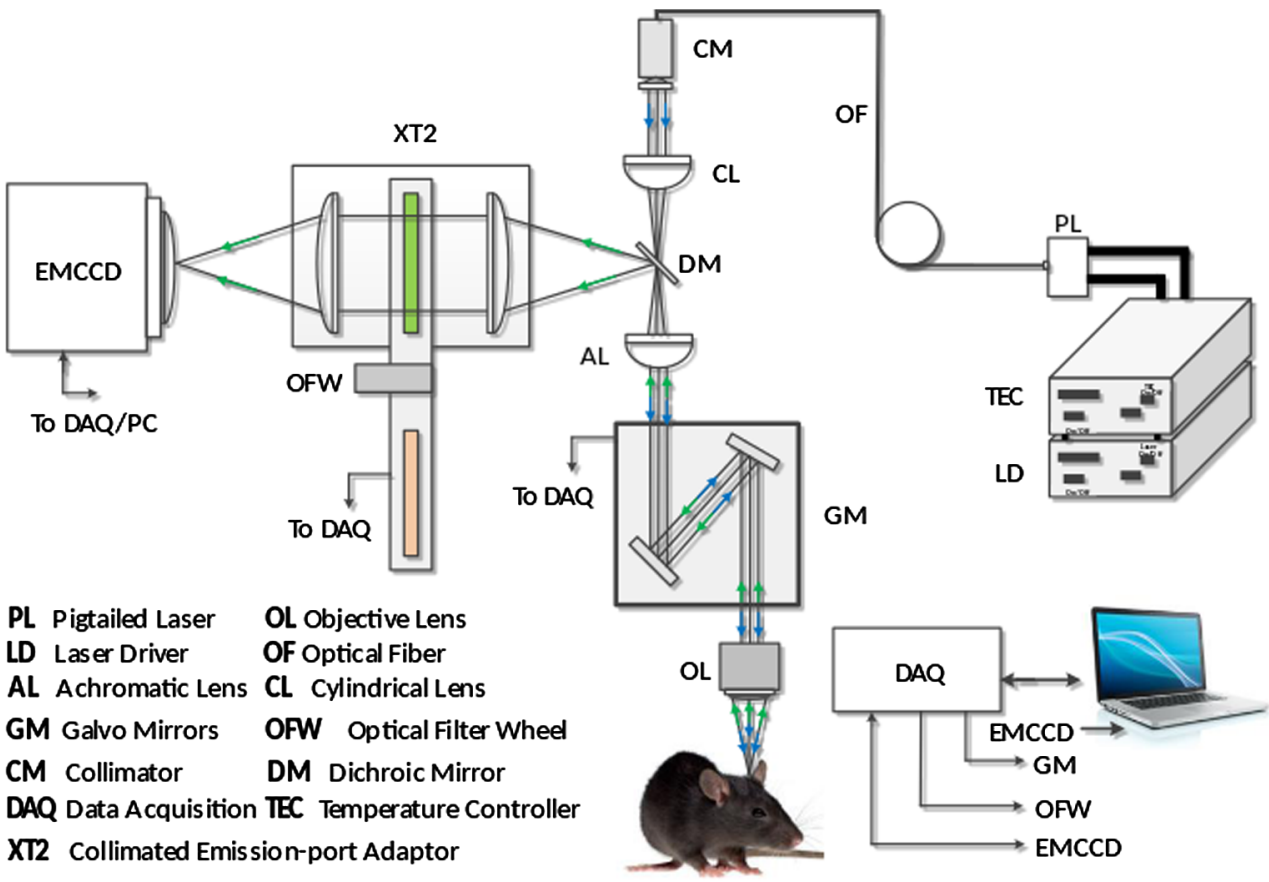

(b)
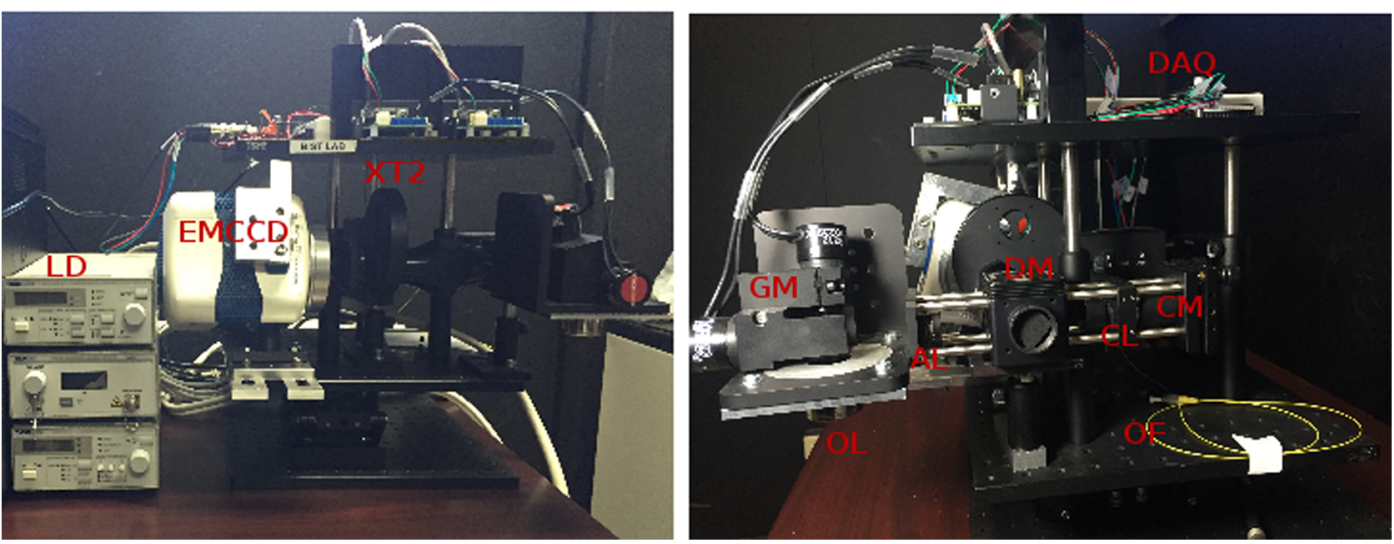

Fig. 2 (a) Block diagram of the FLOT system. Collimated laser light passes through a cylindrical lens for line illumination. A dichroic mirror separates the illumination from the imaging path. Excitation light is focused on the sample by a scan-lens. A pair of galvanometers is used to scan and descan the tissue. The emitted light is reflected by the dichroic beam splitter and passes through a narrow-band emission optical filter and then imaged on the EMCCD sensor. (b) Snapshots of the developed FLOT system.

change in the focal plane caused by the thickness of the filter glass, a two-piece collimated emission-port adapter (XT2, Photometrics, Tucson, Arizona) is used to provide a collimated (infinity corrected) space for a filter wheel. Finally, a highly sensitive electron multiplication charge coupling device (EMCCD) (Evolve 512, Photometrics, Tucson, Arizona) is used to acquire data along the lines parallel to the illumination pattern at each step. Therefore, in this configuration, photons that travel through various depths are detected simultaneously in each single frame captured by the camera.

\subsection{Forward Model for Light Propagation}

Design and implementation of any optical tomography system requires a mathematical forward model, describing light propagation in the medium, and an inverse problem solver to reconstruct unknown parameters by fitting the measurements to the outcome of the forward model. Therefore, modeling and simulation of photon propagation in the medium is an important first step toward any optical device design.

Propagation of photons inside biological tissue can be modeled by the radiative transport equation (RTE). However, the RTE is difficult to solve numerically and exact solutions only exist for relatively simple geometries. The RTE is simplified by applying a set of approximations to take the form of a diffusion equation. In the diffusion approximation regime, it is assumed that the radiation in the medium is almost isotropic; therefore, this model is not suitable for small source-detector separation setups like the proposed FLOT scanner, where the distance between a source and a detector can be less than the photon scattering length. In FLOT setups, Monte Carlo (MC) simulations ${ }^{14}$ are employed to generate the sensitivity matrix for each source-detector pair. ${ }^{5}$ A typical simulated sensitivity matrix for multiple source-detector separations in a phantom 

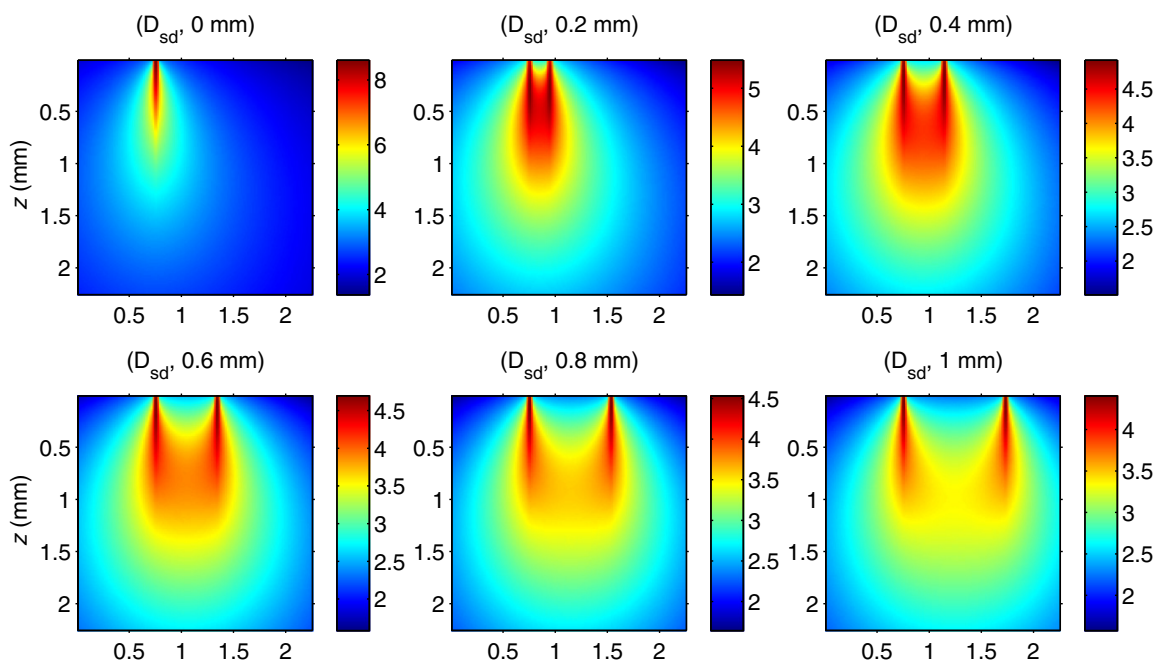

Fig. 3 Simulated sensitivity matrix using MC light propagation mode ${ }^{14}$ for six different source-detector separations in a scattering medium with the optical properties: $\mu_{\mathrm{a}}=1 \mathrm{~mm}^{-1}, \mu_{\mathrm{s}}=9 \mathrm{~mm}^{-1}$, and $g=0.9$.

with optical properties of absorption coefficient $\mu_{\mathrm{a}}=1 \mathrm{~mm}^{-1}$, scattering coefficient $\mu_{\mathrm{s}}=9 \mathrm{~mm}^{-1}$, and anisotropic factor $g=$ 0.9 is shown in Fig. 3.

\subsection{Inverse Problem}

The 3-D distribution of the fluorescent concentration in samples was determined by solving the following equation:

$$
\left(\begin{array}{c}
\mathbf{M}_{11} \\
\mathbf{M}_{21} \\
\vdots \\
\mathbf{M}_{m 1}
\end{array}\right)=\left(\begin{array}{cccc}
W_{11} & W_{12} & \cdots & W_{1 n} \\
W_{21} & W_{22} & \cdots & W_{2 n} \\
\vdots & \vdots & \ddots & \vdots \\
W_{m 1} & W_{m 2} & \cdots & W_{m n}
\end{array}\right) \times\left(\begin{array}{c}
\mathbf{C}_{11} \\
\mathbf{C}_{21} \\
\vdots \\
\mathbf{C}_{n 1}
\end{array}\right)
$$

where the vector $\mathbf{M}$ is determined by the measurements, each row of $W$ represents the voxelized sensitivity matrix for each measurement simulated by using the $\mathrm{MC}$ algorithm, and the vector $\mathbf{C}$ is the spatial distribution of the fluorophore concentration in each voxel. The tomography reconstruction problems are usually ill-posed since the number of measurements is by far less than the number of unknowns. Different analytical and numerical algorithms are used for image reconstruction applications including singular value decomposition method, least square method (LSQR), ${ }^{15}$ algebraic reconstruction technique (ART) ${ }^{16}$ simultaneous iterative reconstruction technique (SIRT), ${ }^{17}$ and simultaneous algebraic reconstruction technique (SART). ${ }^{18} \mathrm{In}$ this work, we employed the SART algorithm for the image reconstruction process since this method benefits from the fast convergence property of the ART and the reconstruction quality of the SIRT algorithm.

One shortcoming in most optical tomography systems is that the sensitivity of the measurements drops exponentially as a function of the photon penetration depth. The result of lower sensitivity at larger depths is the inaccuracy in depth localization where the reconstruction methods are biased to move the objects toward the superficial areas. ${ }^{19,20}$ In Ref. 20, a specific depth compensation algorithm is proposed, which operates to counterbalance this exponential decay of light penetration by modifying the elements of the weight matrix $W$. To apply this compensation algorithm, we assume that the image to be reconstructed is made from $l$ separate layers. In this case, the sensitivity matrix $W$ can be decomposed to $l$ submatrices

$W=\left[W_{1} W_{2}, \ldots, W_{l}\right]$

where $W_{1}$ represents the most superficial layer and $W_{l}$ is the deepest layer. Then the maximum singular value for each submatrix is calculated to generate the matrix $S$, where

$S=\operatorname{diag}\left[M\left(W_{l}\right), M\left(W_{l-1}\right), \ldots, M\left(W_{2}\right), M\left(W_{1}\right)\right]^{\gamma}$.

In this equation, $M\left(W_{j}\right)$ is the maximum singular value of the layer $j$, and $\gamma$ is an adjustable power between 0 and $3,{ }^{20}$ which controls the compensation degree in matrix $S$. In this work, we used $\gamma=1.6$ since it was shown that this value yields the optimal compensation in depth localization. Finally, the sensitivity matrix $W$ was multiplied by matrix $S$ to compensate the decay in the measurement sensitivity as a function of depth. Following this protocol, we use the modified sensitivity matrix $W^{\#}$ defined by

$W^{\#}=W S$.

Based on Eqs. (3) and (4), the maximum singular values in matrix $S$ are arranged inversely from the top to bottom layers while multiplying by the sensitivity matrix $W$, which compensates the decay in the measurement sensitivity as a function of depth.

\subsection{Phantom Fabrication and Calibration Procedures}

To evaluate the performance of the system, we needed a phantom with the optical properties close to brain tissue. Based on our previous experiments, ${ }^{21}$ for rat cortical tissue, typical optical properties are $\mu_{\mathrm{a}}=0.05 \mathrm{~mm}^{-1}, \mu_{\mathrm{s}}=11 \mathrm{~mm}^{-1}$, and $g=0.9$ at 450-nm wavelength. We decided to make silicone-based microfluidic tissue phantoms, which are known to be robust, cost effective samples that have a long shelf life. Using microfluidic fabrication technology, we embedded a microchannel structure inside the phantom, which was then filled with fluorescence 
(a)

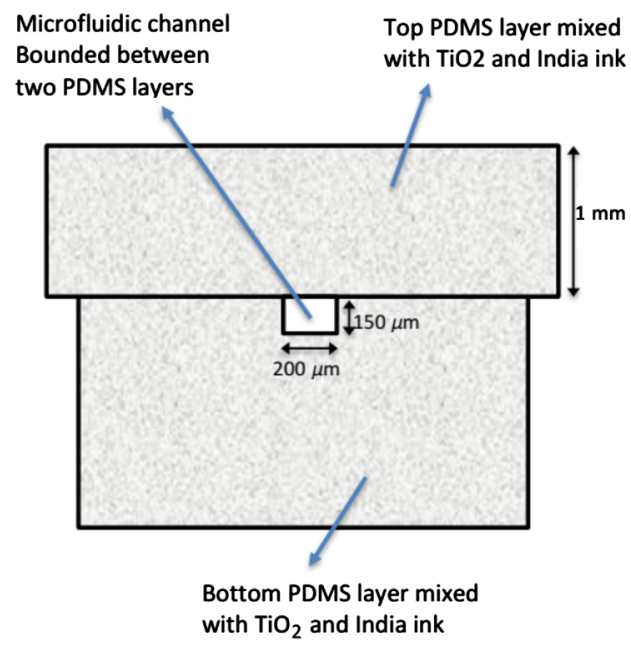

(b)

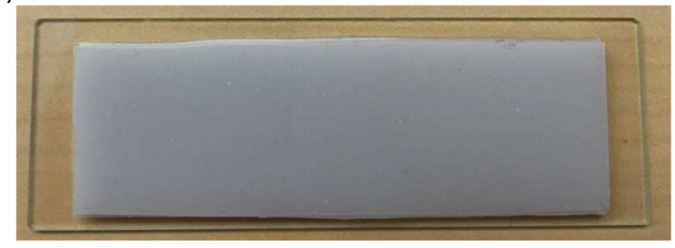

(c)

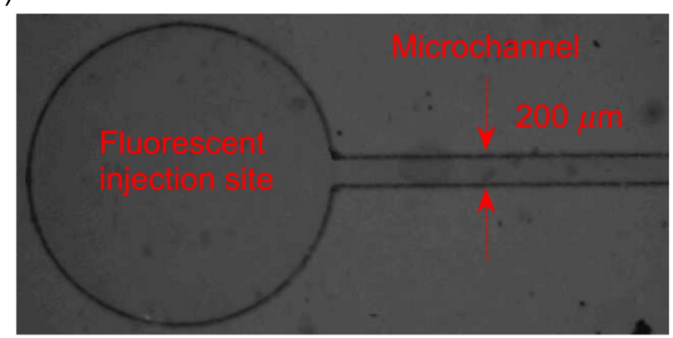

Fig. 4 (a) Cross section of the microfluidic phantom, a channel with the size of $(200 \mu \mathrm{m} \times 150 \mu \mathrm{m})$ is embedded at the depth of $1 \mathrm{~mm}$, (b) and (c) final phantom and its image under the microscope. The channel was filled with FAD solution as fluorescent liquid using a syringe pump.

solutions. Since the channel was embedded within the substrate, there was no need to add extra components to hold the channel.

We fabricated our phantoms in pure polydimethylsiloxane (PDMS) and its associated curing agent (Sylgard 184 silicone elastomer kit, Ellsworth Adhesives, Germantown, Wisconsin) following a 10:1 mixing ratio, to obtain a solid yet flexible structure. Titanium dioxide $\left(\mathrm{TiO}_{2}\right.$, Sigma Aldrich, St. Louis, Missouri) and India ink (Chartpak Inc., Leeds, Massachusetts) were added to this substrate to introduce appropriate amounts of scattering and absorption, respectively. The first step in PDMS molding is the mold design. Such molds come in a variety of shapes and sizes and are designed and fabricated using the soft lithographic method. The molds we used had rectangular channels with different widths and heights. The master for replica molding of the PDMS structure was fabricated via photolithography on a silicon wafer using negative photoresist (SU-8, Microchem Co., Newton, Massachusetts). ${ }^{22}$

Details of the microfabrication process are discussed in Refs. 22 and 23. The surface conditioning of the mold is an important factor in preventing PDMS from sticking. After preparing the mixture, it was poured into the molds and placed into a vacuum chamber for at least $30 \mathrm{~min}$ to remove entrapped bubbles. Next, the prepared mold was placed on a level surface in a $55^{\circ} \mathrm{C}$ heated chamber (oven) for $8 \mathrm{~h}$. After demolding the phantom and punching the holes for the inputs/outputs of the channels, the PDMS structure was treated by a plasma generator (Electro-Technic Products Inc., Chicago, Illinois) and attached to another PDMS layer for permanent bonding without the use of any intermediary contact gel or air gaps. Figure 4 shows the final phantom and a microscopic image of the buried microchannel in PDMS substrate.

Optical properties of the prepared phantom were measured using a double-integrating sphere setup. ${ }^{24,25}$ The schematic of the setup is shown in Fig. 5. The main variables which are measured through these experiments are the diffused reflectance $\left(M_{R}\right)$ and transmittance $\left(M_{T}\right)$, defined by the amount of light reflected by and transmitted through the sample, normalized to the intensity of the incoming light. We used the following equations to calculate these variables: ${ }^{24}$

$$
\begin{aligned}
M_{R} & \equiv r_{\mathrm{std}} \cdot \frac{R\left(r_{s}^{\mathrm{direct}}, r_{s}\right)-R(0,0)}{R\left(r_{\mathrm{std}}, r_{\mathrm{std}}\right)-R(0,0)}, \\
M_{T} & \equiv \frac{T\left(t_{s}^{\mathrm{direct}}, r_{s}\right)-T_{\mathrm{dark}}}{T(0,0)-T_{\mathrm{dark}}} .
\end{aligned}
$$
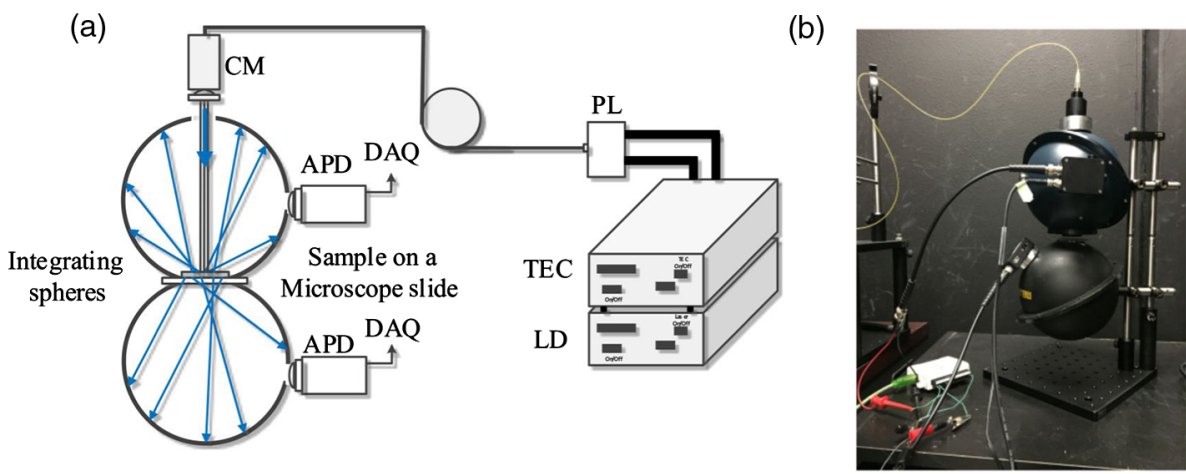

Fig. 5 (a) Schematic of the double-integrating sphere setup used to measure the optical properties of the microfabricated tissue phantoms. (b) A snapshot from the double-integrating sphere optical setup. 

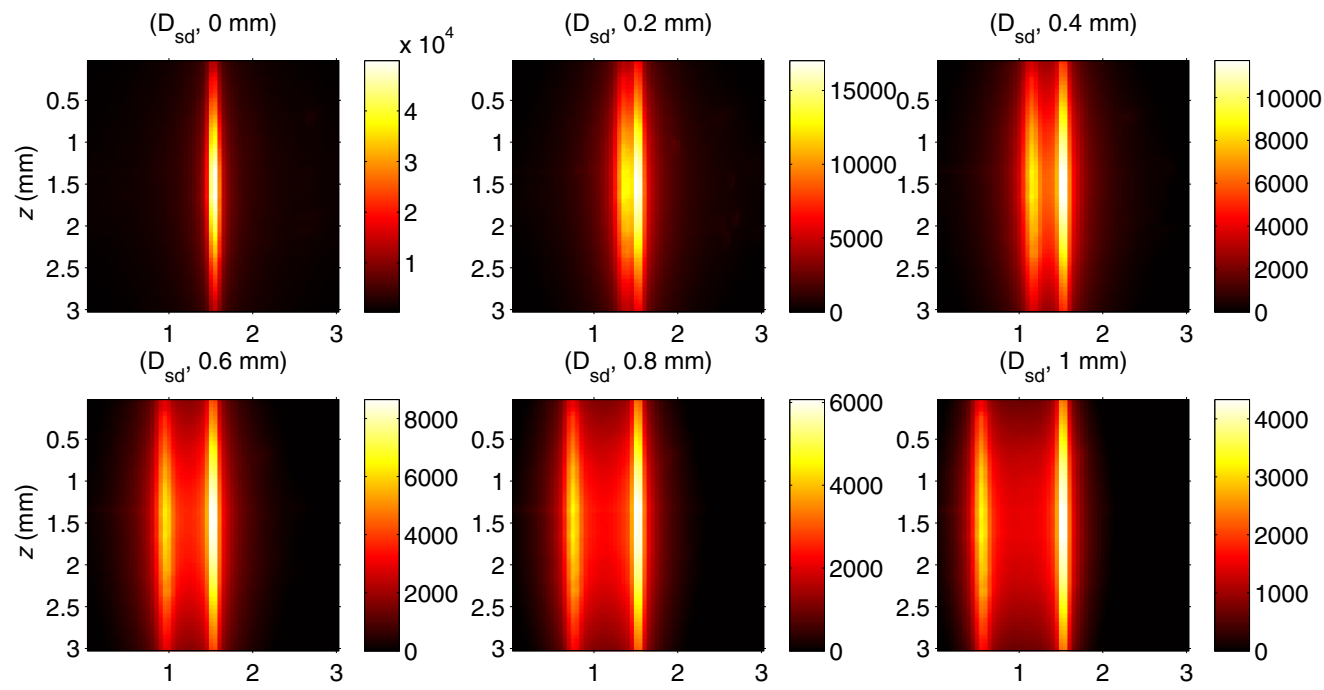

Fig. 6 Experimental raw data obtained by the detectors number 1 to 6 for a microchannel phantom with a rectangular cross section $(200 \mu \mathrm{m} \times 25 \mu \mathrm{m})$ embedded at the depth of $800 \mu \mathrm{m}$.

In these equations, variables $R(0,0), T(0,0)$, and $T_{\text {dark }}$ are calibration measurements, $R\left(r_{s}^{\text {direct }}, r_{s}\right)$ is the diffuse reflected light intensity measured using the photodetector mounted on the top sphere, $T\left(t_{s}^{\text {direct }}, r_{s}\right)$ is the diffuse transmitted light intensity measured by the second photodetector which is installed on the bottom sphere, and $r_{\text {std }}$ is the wall reflectance coefficient of the integrating spheres. ${ }^{25} \mathrm{We}$ employed the inverse adding doubling (IAD) algorithm ${ }^{24}$ to reconstruct the optical properties of the sample using the collected experimental data. The IAD method is an iterative algorithm designed to extract the optical properties
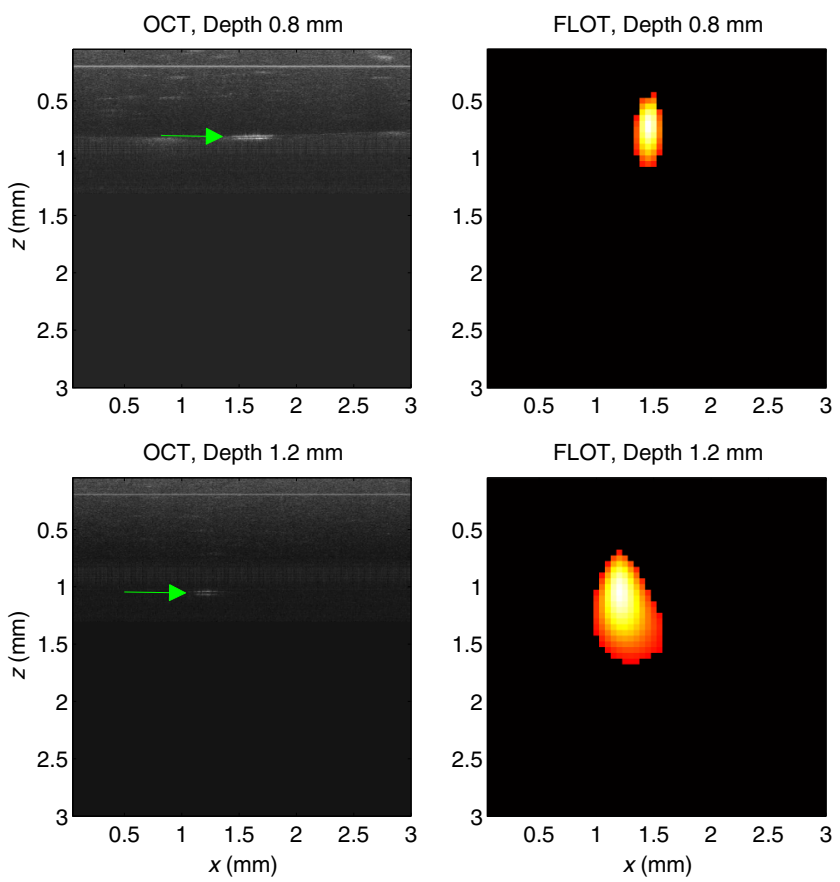

Fig. 7 The reconstruction results of two phantoms, one with the channel size of $(200 \mu \mathrm{m} \times 25 \mu \mathrm{m})$ at the depth of $800 \mu \mathrm{m}$, and one with the channel size of $(150 \mu \mathrm{m} \times 25 \mu \mathrm{m})$ at the depth of $1.2 \mathrm{~mm}$. The exact depth of the microchannel was measured by the OCT system and is shown on the left panel for comparison. A 2-D ZX cross section of the reconstructed channel by SART algorithm is shown on the right panel. of a homogeneous sample from the reflectance and transmittance measurements discussed earlier. The iterations are initiated by assigning some random values to the optical properties of the medium. Then, in each iteration, the algorithm solves the RTE ${ }^{26}$ to calculate the transmission and reflection of the sample and compares these results with the experimental data. Next, it readjusts the values of the optical properties to minimize the error between the calculations and experimental results and continues this process until convergence.

\subsection{Surgical Procedures for In Vivo Experiments}

We used rats to run a sequence of in vivo experiments. All procedures were carried out in the facility accredited by the Association for Assessment and Accreditation and Laboratory Animal Care and approved by the University of Wisconsin-Milwaukee

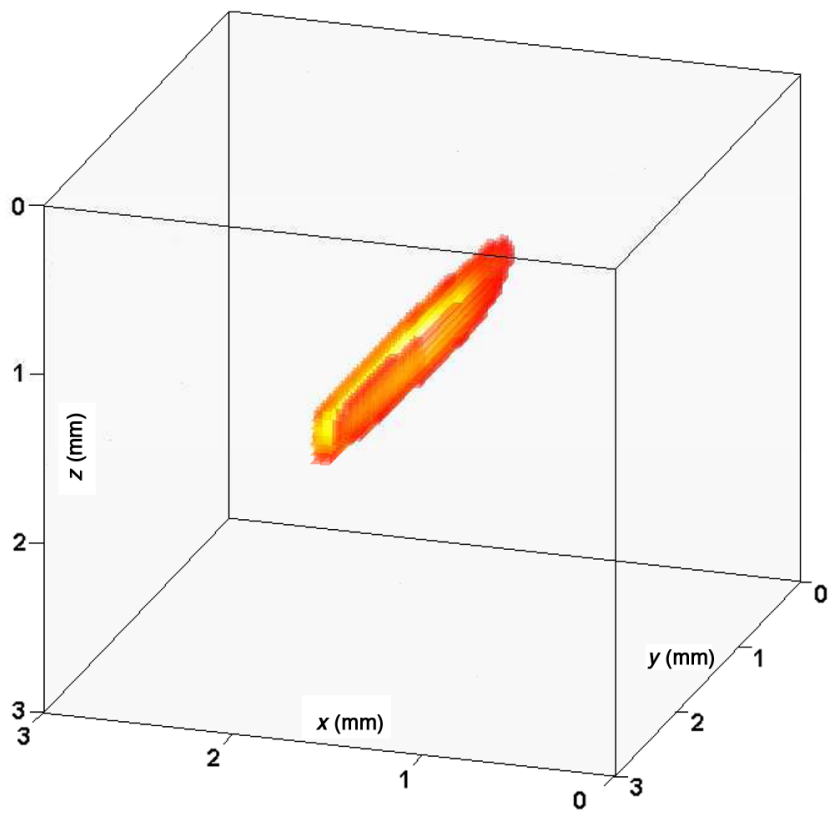

Fig. 8 The 3-D reconstructed image of a microchannel at the depth of $1200 \mu \mathrm{m}$ filled with FAD solution. 


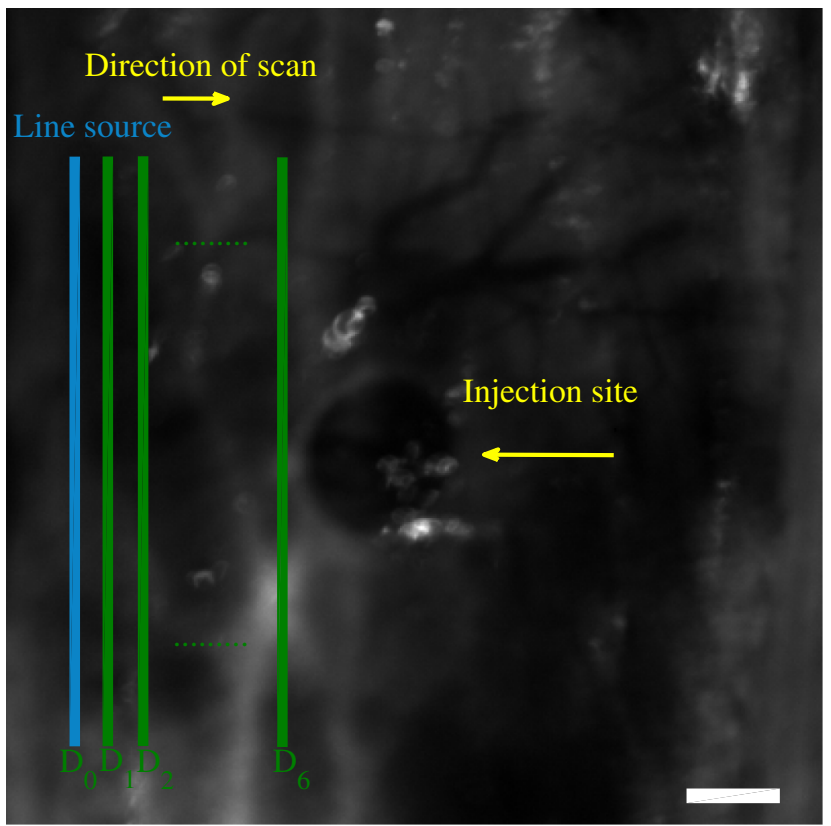

Fig. 9 In vivo experiments were conducted through a thinned-skull rat brain transfected with GFP. The returning fluorescent light was captured by the successively defined detectors $\left(D_{0}\right.$ to $\left.D_{6}\right)$ while the laser beam scans the tissue (scale bar is $500 \mu \mathrm{m}$ ).

Institutional Animal Care and Use Committee and conducted within the ethical guidelines of the National Institutes of Health.

One to four weeks before the experiments, animals were anesthetized with isoflurane in $100 \%$ oxygen (induction occurred with $4 \%$ isoflurane and maintained with $2 \%$ ). During experiments, the head of the animal was fixed in a stereotaxic apparatus. For viral transfection, purified AAV9-CAG-GFP vector was infused into the primary somatosensory cortex (forelimb) $(\mathrm{A} / \mathrm{P}-1.0 \mathrm{~mm}, \mathrm{M} / \mathrm{L}+3.8 \mathrm{~mm})$ and the primary visual cortex (A/P $-5.3 \mathrm{~mm}, \mathrm{M} / \mathrm{L}-3.8 \mathrm{~mm})$. The depth of injection was systematically varied to include $0.8,1.0,1.5$, and $2.0 \mathrm{~mm}$. The injection volume was also systematically varied to include $0.2,0.5$, and $0.75 \mu \mathrm{l}$. The virus was injected at the rate of $0.05 \mu \mathrm{l} / \mathrm{min}$ by using a $10 \mu \mathrm{l}$ syringe and a 34 -gauge needle mounted on the stereotaxic automated injector. The injector was left in place for $10 \mathrm{~min}$ following the injection to allow the diffusion of the virus away from the injector. 1 to 4 weeks later, animals were ready to perform the experiments.

Immediately following the completion of experiments, animals were deeply anesthetized with isoflurane, transcardially perfused with 0.2 MPBS followed by $10 \%$ buffered formalin. Brains were removed and postfixed in $10 \%$ formalin for $24-\mathrm{h}$ and then transferred to $30 \%$ sucrose/PB. The brains were then frozen, sectioned into coronal slices of $200-\mu \mathrm{m}$ thickness, then mounted on glass slides, and coverslipped with UltraCruz mounting medium (Santa Cruz, California) containing $1.5 \mu \mathrm{g} / \mathrm{ml}$ DAPI (for nuclear counter staining).

\section{Experimental Results}

\subsection{Phantom Results}

After fabricating tissue phantoms, flavin adenine dinucleotide (FAD) fluorescent solution was injected into the channel using a syringe pump (Genie Plus, Kent Scientific Corporation, Torrington, Connecticut). Then an area of $3 \mathrm{~mm} \times 3 \mathrm{~mm}$ was scanned by a line-shaped laser beam at $450-\mathrm{nm}$ wavelength, with a resolution of $70 \mathrm{lp} / \mathrm{mm}$. During each illumination, one image was captured by the EMCCD camera and transferred to a computer for further processing and extracting the information of different detectors. A total measured dataset of $200 \times 200 \times 7$ voxels was acquired by the system and nearest-neighbor interpolation
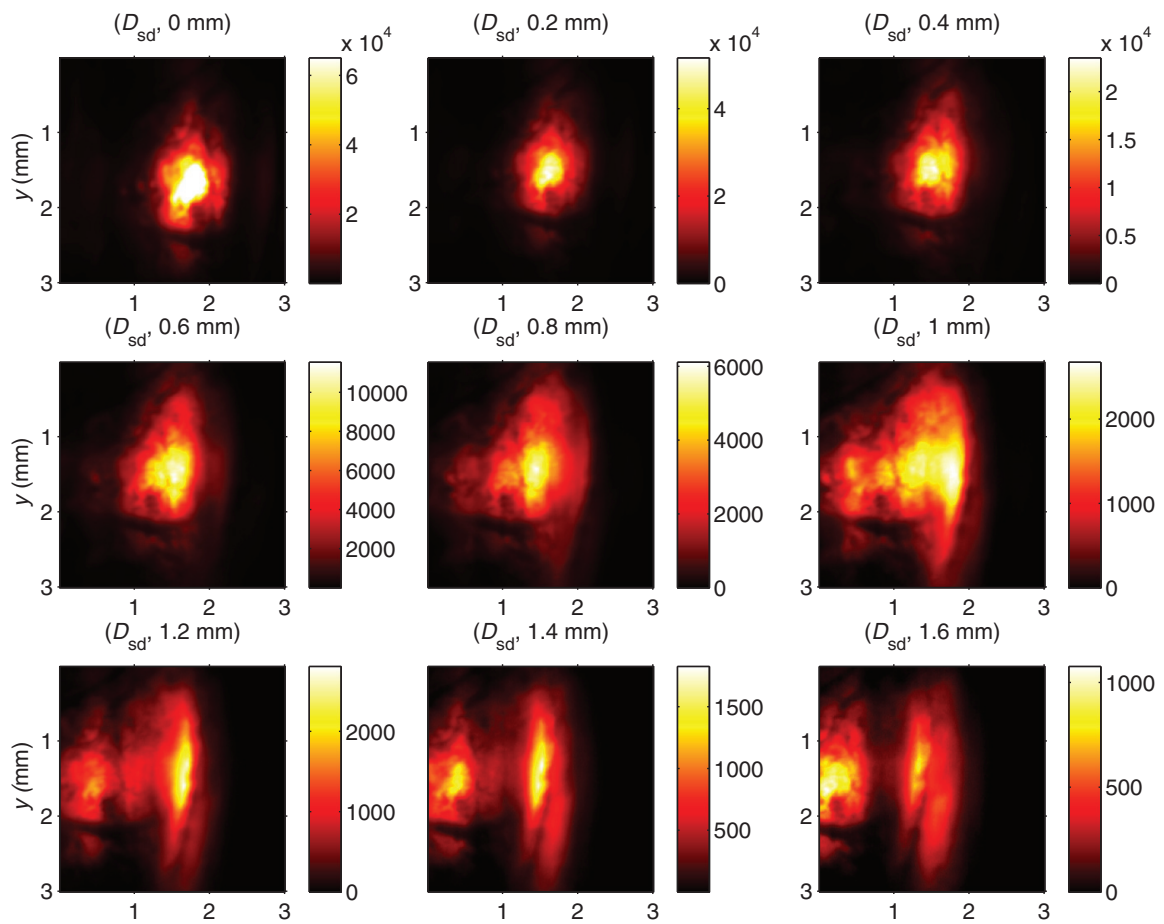

Fig. 10 Experimental raw data of an in vivo scan of a rat cortex through thinned skull, obtained by detectors number 1 to 9 . The injection was performed in the right hemisphere, at the depth of $800 \mu \mathrm{m}$, with GFP. 
method was employed to down-sample it to $60 \times 60 \times 7$ voxels, which was used as the input for our reconstruction algorithm.

As an example, for a microchannel buried at a depth of $800 \mu \mathrm{m}$, the experimental raw data obtained by detectors number 1 to 6 is shown in Fig. 6. This raw data were fed into our reconstruction algorithm to generate the image of fluorescent distribution. The reconstruction was performed in 2-D planes and then a 3-D structure of the fluorescent object was obtained by stacking all the 2-D images together. ${ }^{13}$ The reconstruction process of one 2-D cross section, after 3000 SART iterations, takes $\sim 20 \mathrm{~s}$, when the program is run by a 64-bit operating system, Windows 7 home premium, 8GB RAM, Intel core(TM)i3 $\mathrm{CPU}$ at $3.3 \mathrm{GHz}$.

To find the exact depth of the channel and confirm the reconstruction results, we scanned our sample by a spectraldomain optical coherence tomography (SD-OCT) system, which was developed and tested in the lab prior to current experiments. $^{27,28}$ As displayed in Fig. 7, the OCT images (left panel) show the actual depth of two microchannels buried at depths of 800 and $1200 \mu \mathrm{m}$, and the right panels show the fluorescent reconstruction results generated by the FLOT system. By increasing the depth of the microchannels, the point spread function of the system, and as a result, the diameter of the reconstructed channel increase.

The full width at half maximum of the reconstructed microchannel along the $x$ - and $y$-dimensions are 250 and $450 \mu \mathrm{m}$ for the channel at the depth of $800 \mu \mathrm{m}$, and 450 and $750 \mu \mathrm{m}$ for the channel at the $1200-\mu \mathrm{m}$ depth. Figure 8 shows the $3-\mathrm{D}$ reconstructed image of the $1200-\mu \mathrm{m}$ depth microchannel filled with the FAD solution.

\subsection{In Vivo Results}

All in vivo experiments were conducted on thinned-skull rats (Fig. 9). Each animal received multiple injection of different volumes to express green fluorescent protein (GFP) in cortical areas at different depths and expression levels. Two different mechanisms were employed to compare the FLOT reconstructed results with the actual GFP distribution. After scanning the tissue and collecting the required measurements for the fluorescent tomography, we used our single optical fiber fluorescence probe system $^{29}$ to clarify the axial distribution of the GFP inside the
(A)

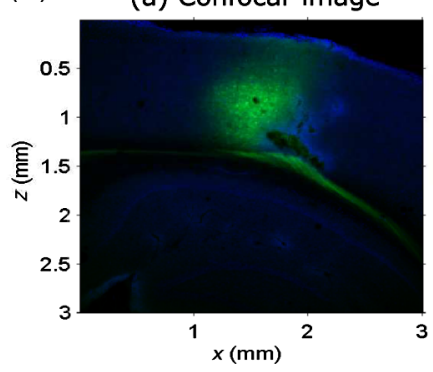

(c) Probe signal

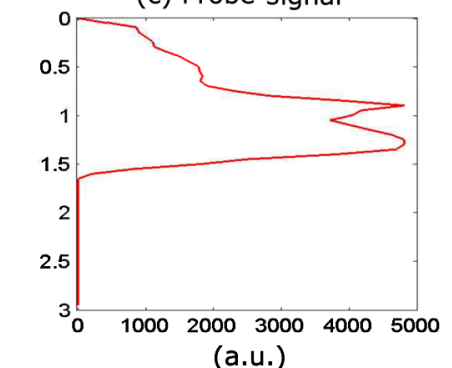

(C)

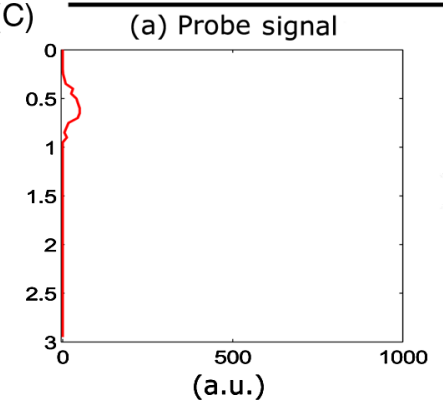

(b) Reconstruction

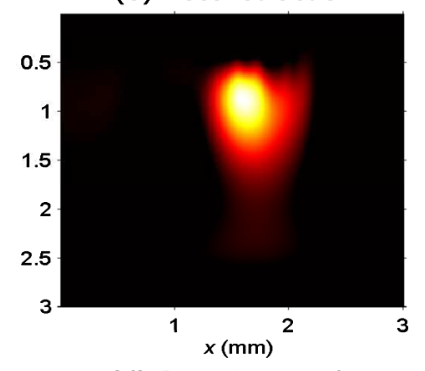

(d) Superimposed

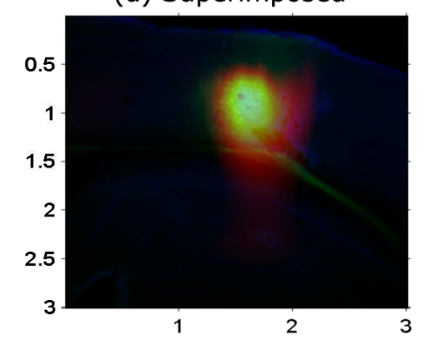

(b) Confocal image
(B)

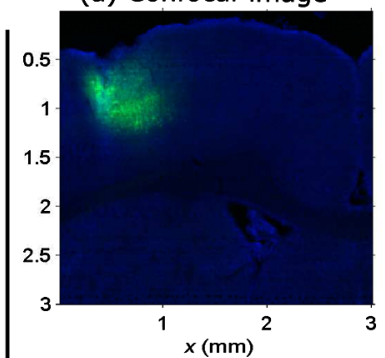

(c) Probe signal

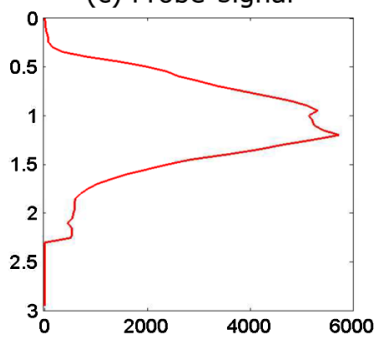

(a.u.) (b) Reconstruction

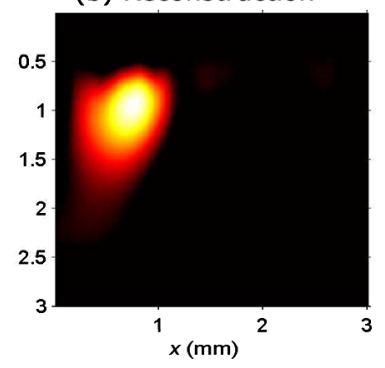

(d) Superimposed

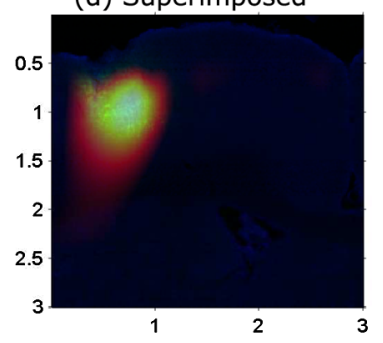

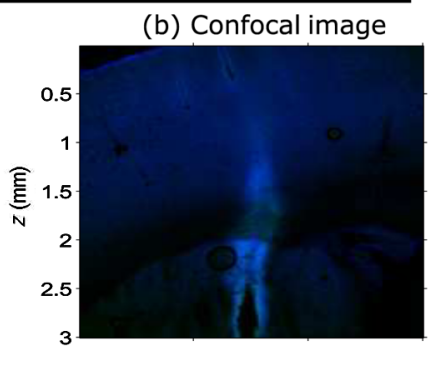

Fig. 11 Experimental results of in vivo scans of rat brains with the injection depth of (A) $800 \mu \mathrm{m}$ in the right hemisphere, (B) $800 \mu \mathrm{m}$ in the left hemisphere, and (C) $800 \mu \mathrm{m}$ in the left hemisphere. In (A) and (B), the gene expression was successful: (a) a confocal microscopy image of a rat brain's slice close to the site of injection, (b) FLOT reconstruction result, (c) curve displays fluorescence signal as a function of depth detected by the single optical fiber probe system, (d) superimposed image of the reconstruction result and the confocal microscopy image. In (C), the gene expression was not successful: (a) curve displays fluorescence signal as a function of depth detected by the single optical fiber probe system, (b) a confocal microscopy image of a rat brain slice close to the site of injection. 

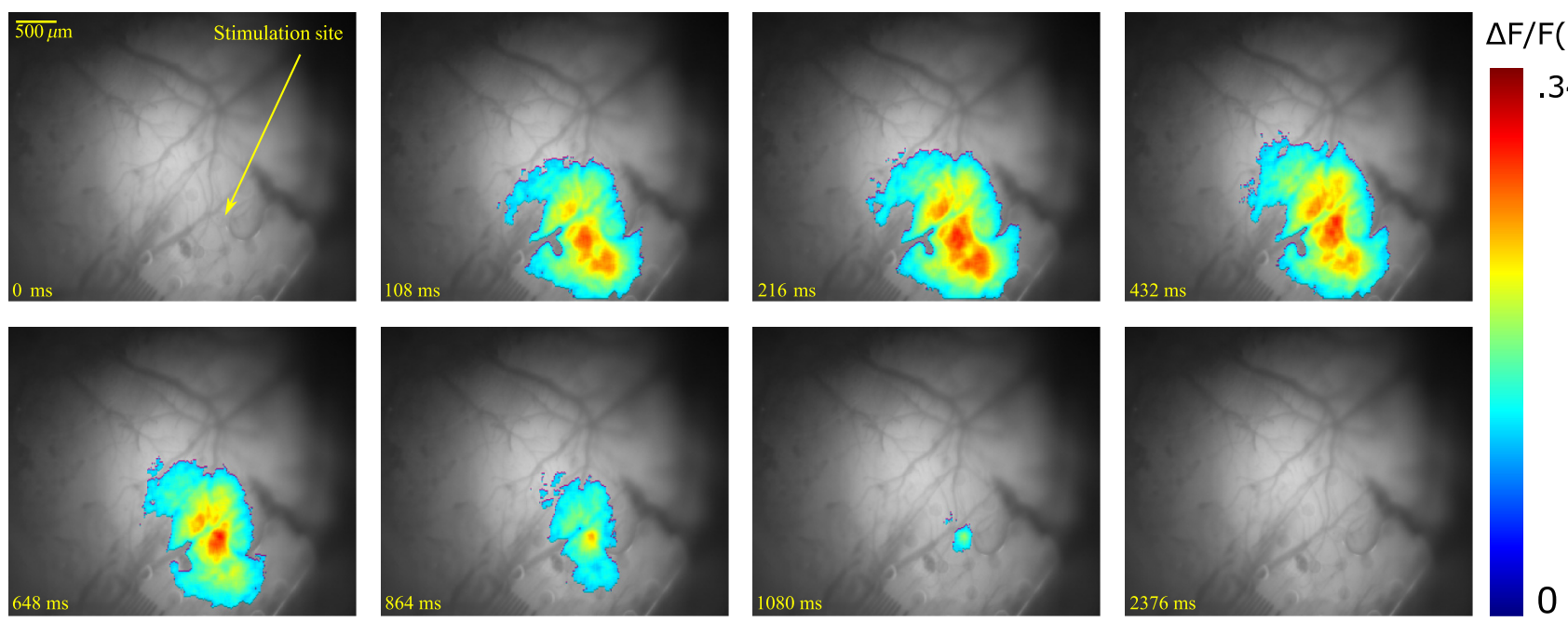

Fig. 12 Spatial and temporal distribution of fluorescent signal due to injection of $100 \mu \mathrm{A}$ electrical stimulation through a graphene array with $150 \mu \mathrm{m}$ site diameter in a transgenic GCaMP6f mouse brain. ${ }^{33}$

brain around the injection site. This system uses a thin multimode optical fiber, which serves as the head of the probe, to deliver blue laser pulses $(473 \mathrm{~nm})$ to the region of interest and guide a sample of the emission signal back to photodetectors. After scanning the tissue with the fiber probe system, the animal was sacrificed and the brain tissue was extracted, sliced, and imaged by a confocal fluorescence microscope to further clarify the real spatial distribution of fluorescence proteins.

Figure 10 shows the experimental raw data collected from the right hemisphere of a rat brain which received injection at the depth of $0.8 \mathrm{~mm}$. For this experiment, the reconstructed images are displayed in Fig. 11(A), where panel (a) shows the confocal image of a rat brain slice with GFP expression. A 2-D cross section of the reconstructed fluorescent distribution is shown in panel (b). Panel (c) shows the normalized axial distribution of GFP recorded by the single fiber probe system and the superimposed image is shown in panel (d) for comparison. Another set of in vivo experimental results is shown in Fig. 11(B), where the rat brain was injected on the left hemisphere at the depth of $800 \mu \mathrm{m}$. In both experiments, the fluorescent reconstruction results are in good agreement with the data from the probe system and also with confocal images.

Despite the fact that all the animals were carefully injected at specific depths with predefined volumes, in a few cases, the gene delivery was not successful and almost no fluorescent signal was detected by the FLOT system. Figure 11(C) shows the experimental results from a case where almost no fluorescent signal was detected by the FLOT system. The data from the fiber probe system and the confocal microscopy images of the brain slices also confirmed that the gene delivery was not successful. These experimental results prove that such a tomography system provides valuable information regarding the depth and level of the expression of optogenetic proteins prior to the experiments without causing any damage to the brain tissue under test.

\section{Conclusion and Future Work}

In this paper, the development of an FLOT system and the corresponding image reconstruction algorithms were discussed in detail and several experimental results were presented in both tissue phantoms and in vivo experiments. In the phantom experiments, the actual depth of the microchannel buried inside a highly scattering medium was determined using a custommade SD-OCT system, ${ }^{27}$ and the reconstruction results using the FLOT system were in reasonably good agreement with the OCT measurements considering the limitations of the method. The in vivo experiments were conducted on thinnedskull rats which were injected to express GFP in the cortical tissue at different depths. Two different mechanisms were employed to confirm the FLOT results. In the first step, a single optical fiber probe system was used to clarify the axial distribution of the GFP at the injection site. Then the brain tissue was sliced and imaged by a confocal microscope. The results from in vivo experiments were quite promising since the FLOT scanner can reveal the 3-D distribution of the GFP within the tissue only by scanning the brain with light from the surface.

Obviously, we expect better results if we use FPs which have red/near-infrared shifted excitation spectra such as tdTomato and mCherry. ${ }^{30,31}$ We are interested in using the developed technology for real-time 3-D monitoring of neural activity following optogenetic/electrode-based stimulation which is currently an active area of research. ${ }^{32}$ To record brain activity and perform optical imaging in parallel, currently, we use our CLEARECoG technology. This technology allows us to perform electrophysiology recording or modulate neural activity as we record hemodynamic signals with SD-OCT system or monitor neural activity via calcium imaging. Figure 12 shows a typical example where the brain tissue is stimulated by injecting current pulses through ECoG electrodes while the induced neural activity is recorded by imaging the fluorescence signal of the genetically encoded calcium indicator GCaMP6f. This calcium indicator has fast kinetics; therefore, 3-D recording of these molecules requires a tomography system that offers fast scanning and recording mechanisms. If the image acquisition time is short enough compared to the physiological fluctuations, time-lapse images can be obtained to draw a clear picture of the dynamics under test and the underlying cellular activity. The proposed FLOT scanner, with some modifications to increase the speed, is an appropriate technology to fulfill the speed requirements for such new applications. 


\section{Disclosures}

There is no competing interest of any kind related to the material of this manuscript.

\section{Acknowledgments}

This research was supported by the Brain and Behavior Research Foundation (NARSAD) young investigator Award Grant No. 20610, and by the National Science Foundation (NSF) Career Award, Award No. 1454300.

\section{References}

1. E. M. C. Hillman, "Optical brain imaging in vivo: techniques and applications from animal to man," J. Biomed. Opt. 12(5), 051402 (2007).

2. Y. Choi et al., "Optical imaging with the use of a scattering lens," IEEE J. Sel. Top. Quantum Electron. 20(2), 61-73 (2014).

3. M. Rajadhyaksha, R. R. Anderson, and R. H. Webb, "Video-rate confocal scanning laser microscope for imaging human tissues in vivo," Appl. Opt. 38(10), 2105-2115 (1999).

4. W. Denk, J. H. Strickler, and W. W. Webb, "Two-photon laser scanning fluorescence microscopy," Science 248(4951), 73-76 (1990).

5. E. M. C. Hillman et al., "Laminar optical tomography: demonstration of millimeter-scale depth-resolved imaging in turbid media," Opt. Lett. 29(14), 1650-1652 (2004).

6. B. Yuan et al., "A system for high-resolution depth-resolved optical imaging of fluorescence and absorption contrast," Rev. Sci. Instrum. 80(4), 043706 (2009).

7. R. Pashaie et al., "Optogenetic brain interfaces," IEEE Rev. Biomed. Eng. 7, 3-30 (2014).

8. K. Deisseroth, "Controlling the brain with light," Sci. Am. 303(5), 48-55 (2010).

9. R. Pashaie and R. Falk, "Single optical fiber probe for fluorescence detection and optogenetic stimulation," IEEE Trans. Biomed. Eng. 60(2), 268-280 (2013).

10. E. M. C. Hillman et al., "Laminar optical tomography: high-resolution 3D functional imaging of superficial tissues," Proc. SPIE 6143, 61431M (2006).

11. E. M.C. Hillman et al., "Depth-resolved optical imaging and microscopy of vascular compartment dynamics during somatosensory stimulation," NeuroImage 35(1), 89-104 (2007).

12. S. Yuan et al., "Three-dimensional coregistered optical coherence tomography and line-scanning fluorescence laminar optical tomography," Opt. Lett. 34(11), 1615-1617 (2009).

13. Y. Chen et al., "Integrated optical coherence tomography (OCT) and fluorescence laminar optical tomography (FLOT)," IEEE J. Sel. Top. Quantum Electron. 16(4), 755-766 (2010).

14. L. Wang, S. L. Jacques, and L. Zheng, "MCML-Monte Carlo modeling of light transport in multi-layered tissues," Comput. Methods Programs Biomed. 47(2), 131-146 (1995).

15. S. M. Stigler, "Gauss and the invention of least squares," Ann. Statist. 9(3), 465-474 (1981).
16. R. Gordon, R. Bender, and G. T. Herman, "Algebraic reconstruction techniques (ART) for three-dimensional electron microscopy and X-ray photography," J. Theor. Biol. 29(3), 471-481 (1970).

17. P. Gilbert, "Iterative methods for the 3D reconstruction of an object from projections," J. Theor. Biol. 36, 105-117 (1972).

18. A. H. Anderson and A. C. Kak, "Simultaneous algebraic reconstruction technique (SART): a superior implementation of the ART algorithm," Ultrason. Imaging 6, 81-94 (1984).

19. V. C. Kavuri et al., "Sparsity enhanced spatial resolution and depth localization in diffuse optical tomography," Biomed. Opt. Express 3(5), 943-957 (2012).

20. H. Niu et al., "Development of a compensation algorithm for accurate depth localization in diffuse optical tomography," Opt. Lett. 35(3), 429-431 (2010).

21. Y. Liu et al., "OptogenSIM: a 3D Monte Carlo simulation platform for light delivery design in optogenetics," Biomed. Opt. Express 6(12), 4859-4870 (2015).

22. T.-W. Lee et al., "Diffusion-based multi-stream bioluminescent reaction in a microfluidic device," Chem. Eng. J. 185-186, 321-327 (2012).

23. F. Ayers et al., "Fabrication and characterization of silicone-based tissue phantoms with tunable optical properties in the visible and near infrared domain," Proc. SPIE 6870, 687007 (2008).

24. S. A. Prahl, M. J. C. van Gemert, and A. J. Welch, "Determining the optical properties of turbid media by using the adding-doubling method," Appl. Opt. 32, 559-568 (1993).

25. M Azimipour et al., "Extraction of optical properties and prediction of light distribution in rat brain tissue," J. Biomed. Opt. 19(7), 075001 (2014).

26. A. Ishimaru, Wave Propagation and Scattering in Random Media, Academic, New York (1978).

27. F. Atry and R. Pashaie, "Analysis of intermediary scan-lens and tube-lens mechanisms for optical coherence tomography," Appl. Opt. 55(4), 646-653 (2016).

28. D. Park et al., "Graphene-based carbon-layered electrode array technology for neural imaging and optogenetic applications," Nat. Commun. 5, 5258 (2014).

29. R. Pashaie and R. Falk, "Single optical fiber probe for fluorescence detection and optogenetic stimulation," IEEE Trans. Biomed. Eng. 60(2), 268-280 (2013).

30. N. C. Shaner, P. A. Steinbach, and R. Y. Tsien, "A guide to choosing fluorescent proteins," Nat. Methods 2(12), 905-909 (2005).

31. N. C. Shaner et al., "Improved monomeric red, orange and yellow fluorescent proteins derived from Discosoma sp. red fluorescent protein," Nat. Biotechnol. 22, 1567-1572 (2004).

32. Q. Tang et al., "In vivo mesoscopic voltage-sensitive dye imaging of brain activation," Sci. Rep. 6, 25269 (2016).

33. S. K. Brodnick et al., "In vivo imaging of spacial and temporal propagation of neural activity after stimulation with a CLEAR microECoG array in GCaMP6f mice," in Proc. of Society for Neuroscience (SFO) (2015).

Biographies for the authors are not available. 